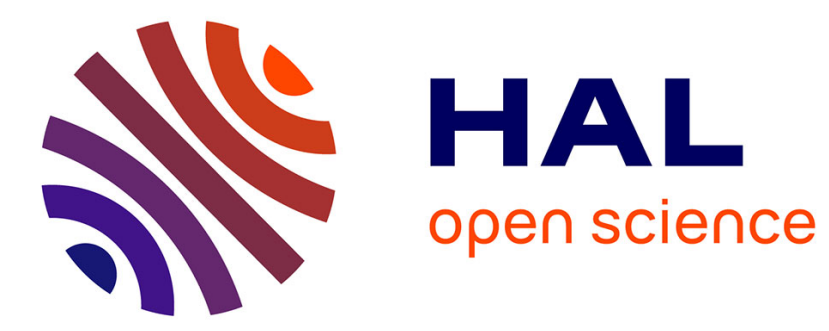

\title{
Micrometric mapping of absolute trapping defects density using quantitative luminescence imaging
}

Gilbert El-Hajje, Daniel Ory, Jean-Francois Guillemoles, Laurent Lombez

\section{To cite this version:}

Gilbert El-Hajje, Daniel Ory, Jean-Francois Guillemoles, Laurent Lombez. Micrometric mapping of absolute trapping defects density using quantitative luminescence imaging. IEEE PVSC, Jun 2016, Orlando, United States. hal-02180990

\section{HAL Id: hal-02180990 https://hal.science/hal-02180990}

Submitted on 11 Jul 2019

HAL is a multi-disciplinary open access archive for the deposit and dissemination of scientific research documents, whether they are published or not. The documents may come from teaching and research institutions in France or abroad, or from public or private research centers.
L'archive ouverte pluridisciplinaire HAL, est destinée au dépôt et à la diffusion de documents scientifiques de niveau recherche, publiés ou non, émanant des établissements d'enseignement et de recherche français ou étrangers, des laboratoires publics ou privés. 


\title{
Micrometric mapping of absolute trapping defects density using quantitative luminescence imaging
}

\author{
Gilbert El-Hajje**1,2,4 , Daniel Ory ${ }^{1,2,4}$, Jean-François Guillemoles ${ }^{2,3,4}$ and Laurent Lombez ${ }^{2,4}$ \\ ${ }^{1}$ EDF R\&D, 6 quai Watier, 78400 CHATOU Cedex, FRANCE \\ ${ }^{2}$ Institute of Research and Development on Photovoltaic Energy (IRDEP), UMR 7174 CNRS-EDF- Chimie \\ ParisTech, EDF R\&D, Chatou, France \\ ${ }^{3}$ NextPV, CNRS-RCAST joint lab, Tokyo University, Tokyo, Meguro-ku 153-8904, Japan \\ ${ }^{4}$ IPVF, Institut Photovoltaïque d'Ile-de-France, 8, rue de la Renaissance - 92160 Antony, France
}

\begin{abstract}
In the present study, we develop a contactless optical characterization tool that quantifies and maps the trapping defects density within a thin film photovoltaic device. This is achieved by probing time-resolved photoluminescence and numerically reconstructing the experimental decays under several excitation conditions. The values of defects density in different $\mathrm{Cu}(\mathrm{In}, \mathrm{Ga}) \mathrm{Se} 2$ solar cells were extracted and linked to photovoltaic performances such as the open-circuit voltage. In the second part of the work, the authors established a micrometric map of the trapping defects density. This revealed areas within the thin film CIGS solar cell with low photovoltaic performance and high trapping defects density. The final part of the work was dedicated to finding the origin of the spatial fluctuations of the thin film transport properties. To do so, we started by establishing a micrometric map of the absolute quasiFermi levels splitting within the same CIGS solar cell, using the hyperspectral imager. A correlation is obtained between the map of quasi-Fermi levels splitting of and the map of the trapping defects density. The latter is found to be the origin of the frequently observed spatial fluctuations of thin film materials properties.
\end{abstract}

Index Terms - CIGS solar cells, time-resolved luminescence, trapping defects quantification, PV performance, carrier dynamics.

\section{INTRODUCTION}

One of the signature traits of CIGS solar cells is the abnormal variations in its photovoltaic performance following the activation of its metastabilities that are present at the absorber surface and its interface with the buffer layer. By using conventional characterization methods, it has been shown[1][2] that metastabilities exist in different categories.

In this work, we aim at developing a contactless optical characterization tool that can quantify and map at the micrometric scale, the defects density that is behind the metastabilities in CIGS devices. In addition, in order to validate our technique we also aim at linking our defects density values to standard PV performance indicators. We will finally attempt to use these defects density maps in order to reveal the origin behind the frequently observed spatial inhomogeneities of thin film solar cells properties.

\section{DESCRIPTION OF APPROACHES \& TECHNIQUES}

To fulfill the main purpose of this study, the potential of high resolution confocal microscopy and that of hyperspectral imaging of photoluminescence emission were combined and the derived results were quantitatively correlated.

On the one hand, the authors develop a system of rate equations that reconstructs an experimental time-resolved photoluminescence (TRPL) decay obtained under pulsed laser excitation. The corresponding trapping defects density is then derived. We show that our technique is robust as the obtained defects density values are constant regardless of the experimental conditions. In addition, the global reconstruction error does not exceed $7 \%$. Our technique is then applied to a set of five CIGS devices on which $\mathrm{J}-\mathrm{V}$ measurements were also made [3]. The trapping states are then correlated to global cell properties.

On the other hand, using spatially and time-resolved photoluminescence, we add the spatial dimension to our method. This resulted in a sub-micron map of the material trapping defects density [4]. This map is compared to a map of absolute values of its quasi-Fermi levels splitting $(\Delta \mu)$ obtained using a hyperspectral imager. The trapping states are then correlated to local cell properties.

\section{EXPERIMENTAL RESULTS \& DISCUSSIONS}

\section{A. Photo-Excitation Power And Voltage-Bias-Dependent TRPL Decays}

In this part of the study, the authors acquired TRPL decays under two different excitation sources. We either vary the incident photo-excitation power, or keep the photo-excitation power constant and inject increasing value of current into the sample. Interestingly, in both cases, the initial double exponential decay becomes increasingly a mono exponential decay of photoluminescence. This can be seen in Figures 1.a \& 1.b: 


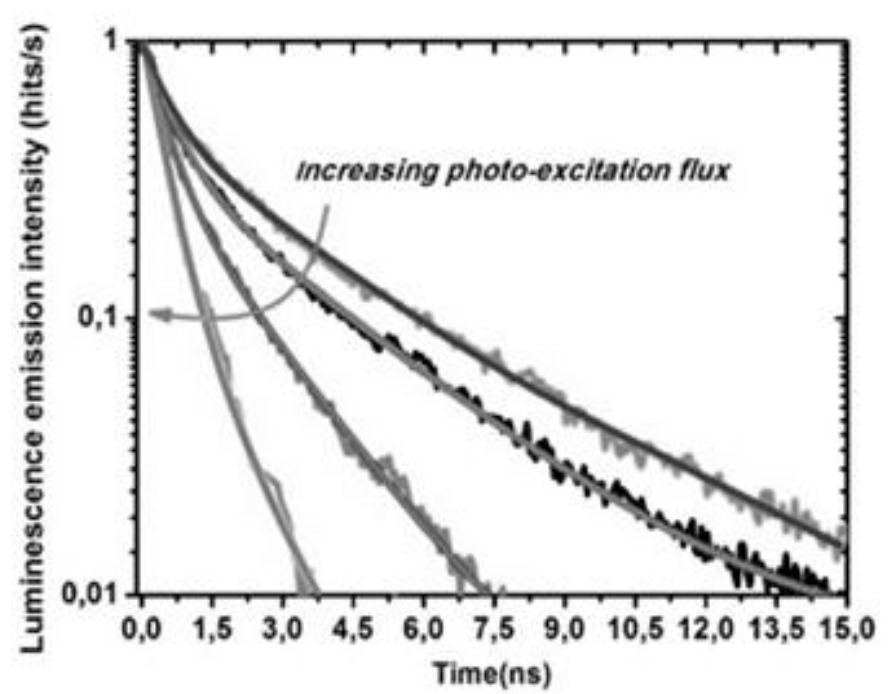

Fig. 1.a. Evolution of the TRPL decays behavior as a function of photo-excitation power.

In our photo-excitation power-dependent TRPL experiments, the value of excitation power is increased. As we will see later, the excess photogenerated carrier density becomes progressively dominant over the accessible trap states density. This increasing ratio between the former and the latter densities is at the origin of the observed convergence from a double to a mono-exponential decay [5].

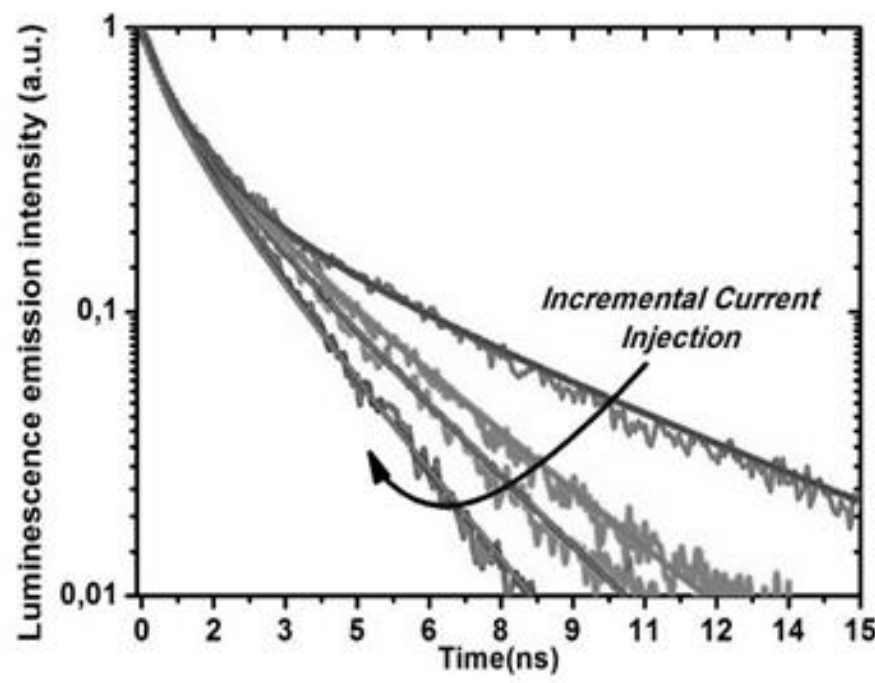

Fig. 1.b. Evolution of the TRPL decays behavior as a function of current injection.

In the voltage-bias-dependent TRPL experiments, the value of the injected current is increased. It tends to electrically occupy the inactive shallow trap states density present at the absorber surface and its interface with the CdS buffer layer. As the latter density decreases, so does the delay in the luminescence emission induced via carrier trapping. This explains why the TRPL decays in Figure 1.b are becoming shorter under an increasing value of injected current.
Finally, whether it is the current injection, or the excitation power that is being increased, the final state regarding the minority carrier dynamics is the same. The latter is characterized by a total dominance of direct recombination events over the trapping events.

\section{B. Quantification Of Trapping Defects Density}

The authors start by a establishing a system of differential rate equations that describes the dynamics of the different populations that are behind the experimental time-resolved luminescence decays. All of the parameters used to solve this system are actual experimental data. Then, an optimization procedure is used in order to reconstruct the experimental time-resolved photoluminescence (TRPL) decay and extract the system trap states density.

The first parameter taken into consideration is the experimental charge carriers' photogeneration. Experimentally, a laser pulse is used in order to induce the photogeneration. Therefore, in order to replicate the experimental excitation conditions of $1.4 \times 10^{13}$ Photons $/$ Pulse $/ \mathrm{cm}^{2}$ delivered within a 6 ps pulse width, the following function is used:

$$
P(t)=\frac{P_{0}}{\left(\cosh \left(\frac{t}{\tau_{p}}\right)\right)^{2}}
$$

$\mathrm{P}(\mathrm{t})$ is the laser pulse temporal profile , $\mathrm{P}_{0}$ is the actual peak power which is equal to $23.5 \mathrm{~mW}$, $\tau \mathrm{p}$ is the laser pulse width which is equal to $6 \mathrm{ps}$.

Three different populations of states are defined here: N1, N2 and N3. N1 is the population occupying the valence band, N3 is the population occupying the conduction band and N2 is the population occupying the accessible shallow trap states density. The densities of accessible states with the $\mathrm{CB}$ and the VB are considered as infinite reservoirs compared to that of the shallow traps which we denote here as $\Psi_{\mathrm{D}}$ and aim to determine its value.

In order to describe the dynamics behind the experimental decays, the system considers three different types of events: Firstly, direct carrier recombination events, such as SRH and band-to-band recombination. The corresponding lifetime $\tau_{\text {Rec }}$ is extracted from the initial fast part of the experimental timeresolved photoluminescence decay. The second and third type of events will be related to the existence of the shallow trap states. In particular, traps capture charge carriers and then emit them by means of thermal activation. Consequently, a capture lifetime $\tau_{\text {Cap }}$ and an emission lifetime $\tau_{\mathrm{em}}$ are defined, and both are considered equal. These lifetimes will be the characteristic lifetimes of the second slower part of the time-resolved photoluminescence decay. 
We propose in Fig.2 a schematic illustration of the studied carrier dynamics:

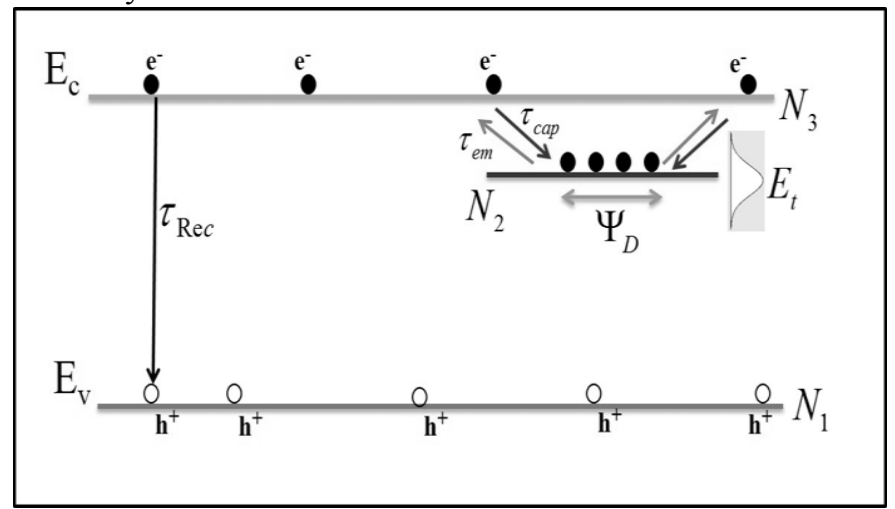

Fig. 2. Schematic illustration of minority carrier dynamics

Now that all of the parameters have been defined, we were able to establish the system of differential rate equations that replicate the experimentally probed carrier dynamics:

$$
\left\{\begin{array}{l}
\frac{d N_{1}}{d t}=+\frac{N_{3}}{\tau_{\operatorname{Rec}}}-G\left(t=\tau_{p}\right) \\
\frac{d N_{2}}{d t}=\left(1-\frac{N_{2}}{\Psi_{D}}\right)\left(\frac{N_{3}}{\tau_{C a p}}\right)-\left(\frac{N_{2}}{\tau_{e m}}\right) \\
\frac{d N_{3}}{d t}=\frac{N_{2}}{\tau_{e m}}-\left(1-\frac{N_{2}}{\Psi_{D}}\right)\left(\frac{N_{3}}{\tau_{C a p}}\right)-\left(\frac{N_{3}}{\tau_{\mathrm{Rec}}}\right)+G\left(t=\tau_{p}\right)
\end{array}\right.
$$

The generation which takes place during $\tau_{\mathrm{p}}$ is written as:

$$
G\left(t=\tau_{p}\right)=P\left(t=\tau_{p}\right)[1-R(\lambda=700 \mathrm{~nm})]
$$

$\mathrm{R}(\lambda=700 \mathrm{~nm})$ is the reflection coefficient that is found to be close to $20 \%$.

In order for the shallow trap states density to be the only unknown parameter, the capture and the emission lifetimes need to be calculated. The values of these lifetimes are dependent on the properties of the material shallow traps. In other terms, one needs to determine the nature of the shallow trap (donor or acceptor), its energy level $E_{t}$ and its capture cross section $\sigma_{\mathrm{e}}$.

The emission lifetime can be calculated as follows:

$$
\frac{1}{\tau_{e m}}=\Psi_{D} \sigma_{e} \nu_{t h} \exp \left(-\frac{E_{t}}{k T}\right)
$$

$v_{\text {th }}$ is the thermal velocity, $\mathrm{k}$ is Boltzmann's constant and $\mathrm{T}$ is the temperature.

The total system of rate equations becomes now a function of only one variable, the trap states density $\Psi_{\mathrm{D}}$.

The numerical procedure consists of varying $\Psi_{\mathrm{D}}$ over a large array of different values. For each value, the system of rate equations in (2) results in a decay $T R L_{N u m}$ that is a reconstruction of the experimental one $T R L_{E x p}$. The optimization procedure then selects the value of $\Psi_{\mathrm{D}}$ for which the reconstruction error is minimized. The latter error is calculated over the whole set of decay points $\mathrm{N}_{\mathrm{p}}$ and is expressed as:

$$
\xi=\frac{\sum_{i=1}^{N_{p}}\left[\left(\frac{\left|T R L_{E x p}(i)-T R L_{\text {Num }}(i)\right|}{T R L_{E x p}(i)}\right) x 100\right]}{N_{p}}
$$

We obtain values of $\xi$ varying between $2 \%$ and $7 \%$. These operations are repeated for all of the experimental decays.

In Fig. 3.a below, we illustrate how the solution of the system of rate equations results in a reconstruction of the experimental TRPL decay. Fig. 3.b illustrates how the reconstruction is made using an array of trapping defects density values and how the optimization algorithm chooses the value that leads to a minimum of reconstruction error. In the present example, the reconstruction error $\xi$ is equal to $5 \%$ and the corresponding $\Psi D$ is equal to $4.7 \times 10^{14}$ traps $/ \mathrm{cm}^{3}$.

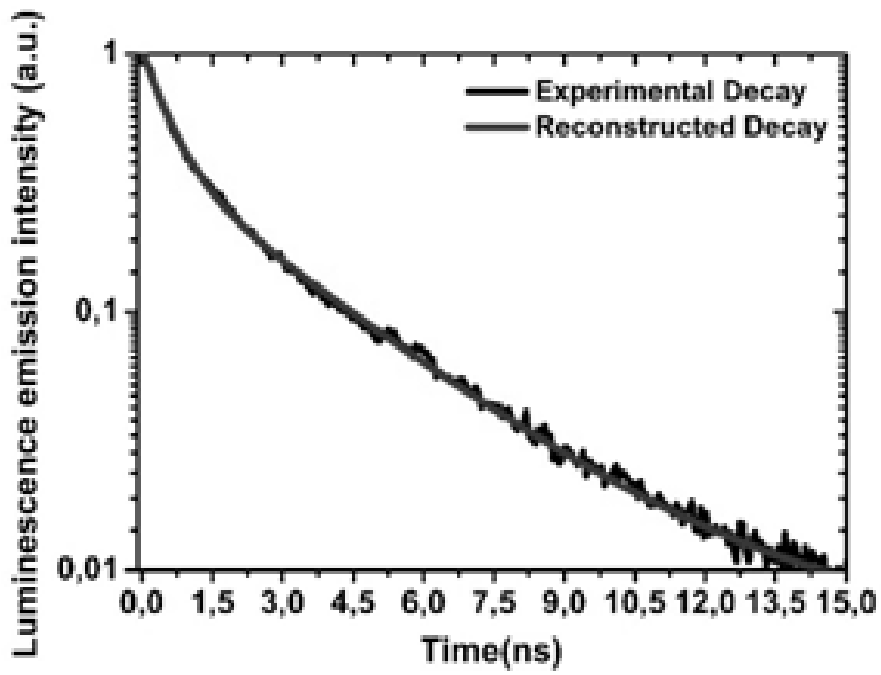

Fig. 3.a. Illustration of a TRPL decay reconstruction

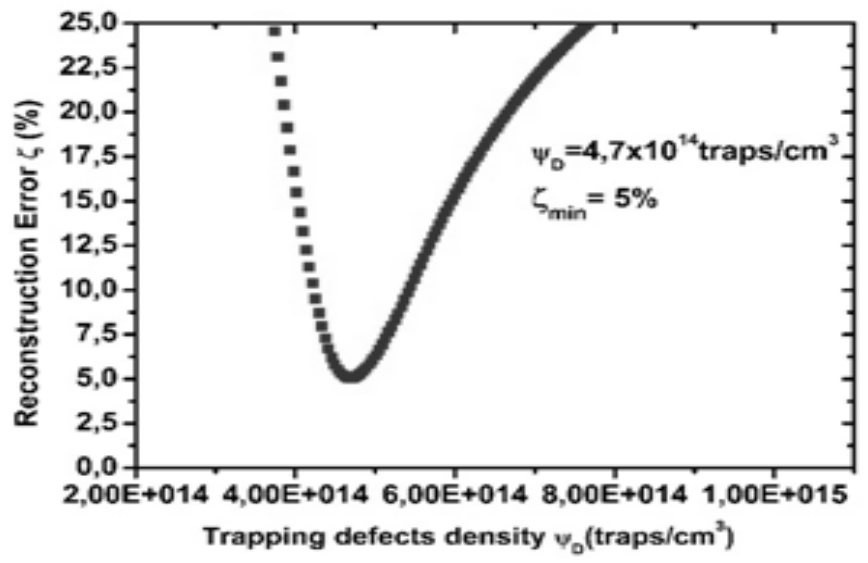

Fig. 3.b. Illustration of the reconstruction error minimization 
For both sets of experiments, we apply our technique and prove that it is self-consistent with respect to a variation of the incident excitation power and the injected current, when the latter are taken into account.

This is proved by the constant value of trapping defects density of $1.055 \times 10^{15} \mathrm{traps} / \mathrm{cm}^{3}$ that it provides, with fluctuations not exceeding $6 \%$ with respect to the mean value.

For the excitation power-dependent TRPL experiments, this means that only one contactless measurement of a TRPL decay, at any given photo-excitation flux, is needed for the calculation of the trapping defects density.

We were so far able to prove that our technique can indeed provide precise reconstructions of the experimental timeresolved luminescence decays and quantify the material trapping defects density.

\section{The effect Of Trapping Defects On Global PV Performances}

At this stage we will test if our defect quantification tool can be used as a reliable indicator of thin film PV solar cells performance. In other terms we will try to evidence the existence of a link between a trap states density derived from TRPL experiments using our method, and the standard PV performances.

To do so, standard $\mathrm{J}(\mathrm{V})$ characterization are performed on five CIGS solar cell devices. All five devices have the same structure and type of layers.

For each device, all of the performance parameters will be extracted. Then, we perform a single acquisition of a TRPL decay on each CIGS device. Using our previously developed method, we finally extract the value of the trap states density within each device.

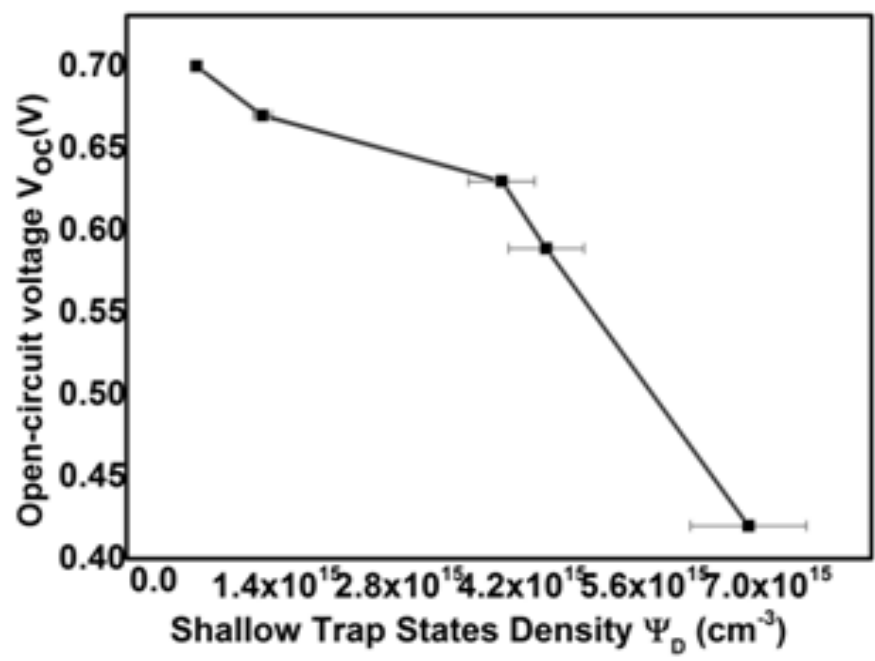

Fig. 4. Evolution of the open-circuit voltage as a function of the shallow trap states density
According Figure 4, it is the open-circuit voltage that revealed a dependence on the device shallow trap states density among all of the standard PV performance parameters.

We deduce that the thin film CIGS device that suffers from the highest trap states density is expected to have the lowest open-circuit voltage. On the one hand these results is in good agreement with the study done in Ref[6] where the highest density of ((In or $\mathrm{Ga}) \mathrm{Cu}$ ) defects ( $(\mathrm{InCu}++$ in our case) induced the lowest Voc.

On the other hand, this validates the type and characteristics of the trapping defects that we are studying here.

For our five CIGS devices, we may not be certain of the exact origins behind each of these different defect densities but we however evidenced their nature, validated their values and their effect on the PV devices performance using a unique approach based on TRPL decays analysis.

\section{The Effect Of Trapping Defects On Local PV Performances}

We start by performing spatially-resolved measurements of the TRPL by scanning the entire CIGS solar cell surface. Then, by applying our technique to each of the TRPL decays, we obtained a complete micrometric map of the trapping defects density. The map exhibited important fluctuations ranging between $3.5 \times 10^{14} \mathrm{~cm}^{-3}$ and $1.05 \times 10^{15} \mathrm{~cm}^{-3}$.

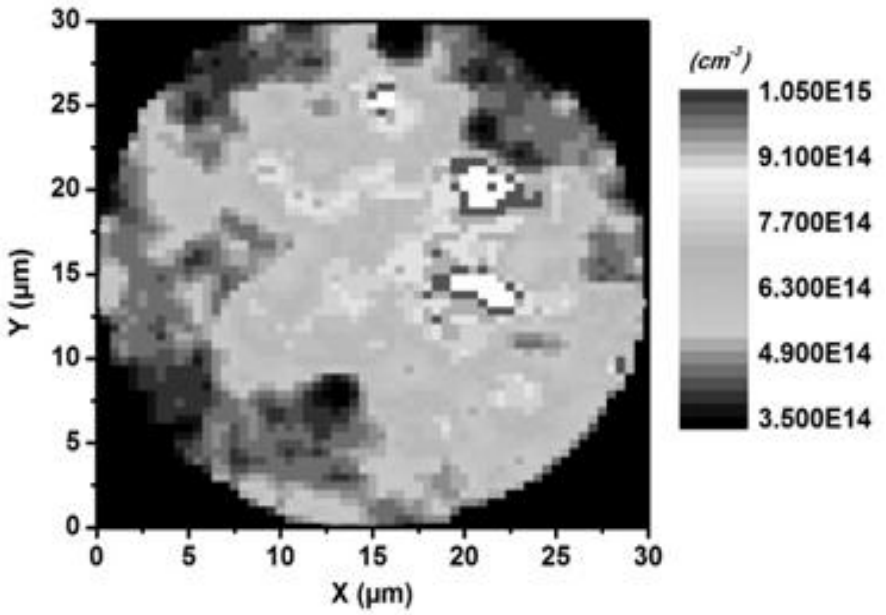

Fig. 5. Micrometric map of the solar cell absolute trapping defects density distriubtion

Finally, based on the hyperspectral imager and the generalized Planck's law for luminescence emission, we establish a map of the absolute value of the quasi-Fermi levels splitting $\Delta \mu(\mathrm{eV})$ within the same solar cell [7]. 


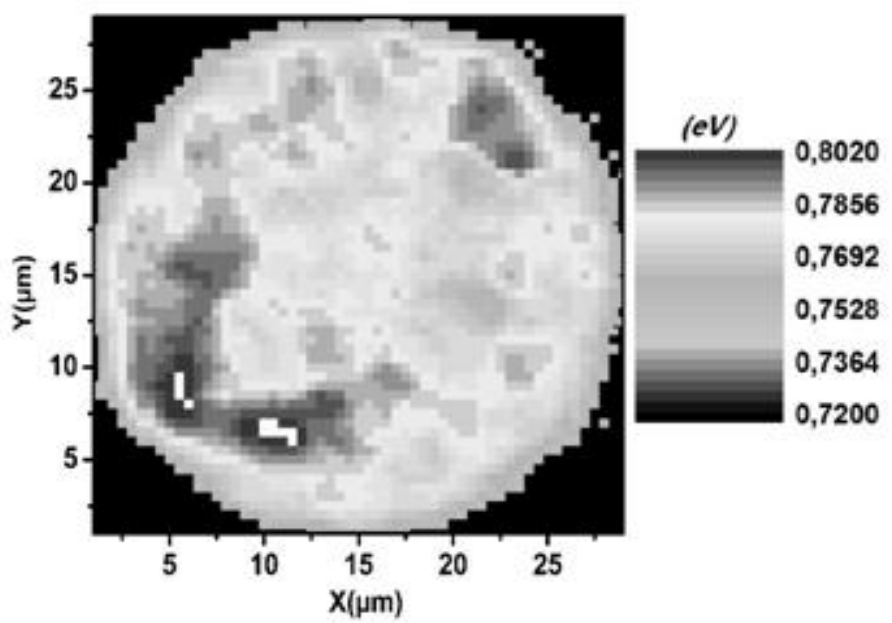

Fig. 6. Micrometric map of the solar cell absolute quasi-Fermi levels splitting

A comparison revealed a dependence of the spatial fluctuations of quasi-Fermi levels splitting in a CIGS solar cell on its trapping defects density. This comes in good agreement with the above results where we evidenced a link between the $\mathrm{V}_{\mathrm{oc}}$ of a solar cell and its trapping defects density. We deduce that the spatial fluctuations of $\Delta \mu$ are dictated by those of the trapping defects density.

This identified link has multiple important implications. First of all, the authors identified at least one of the origins behind the usually observed spatial inhomogeneities of the maximum achievable open circuit voltage of a CIGS solar cell. Second of all, a correlation between the performed experiments is consequently achieved at the global and the micro scale.

\section{REFERENCES}

[1] Müller, T. C. M. et al. "Effect Of Light Soaking On The ElectroAnd Photoluminescence Of $\mathrm{Cu}(\mathrm{In}, \mathrm{Ga}) \mathrm{Se} 2$ Solar Cells". Appl. Phys. Lett. 103.18 (2013): 183504.

[2] Eisenbarth, Tobias et al. "Characterization Of Metastabilities In $\mathrm{Cu}(\mathrm{In}, \mathrm{Ga}) \mathrm{Se} 2$ Thin-Film Solar Cells By Capacitance And Current-Voltage Spectroscopy". J. Appl. Phys. 110.9 (2011): 094506.

[3] El-Hajje, G. et al. "Quantitative luminescence-based measurement of trapping defects density in thin film solar cells". Submitted to Prog. Photovolt: Res. Appl. May 2016.

[4] El-Hajje, G. et al. "On the origin of the spatial inhomogeneity of photoluminescence in thin-film CIGS solar devices". Submitted to Applied Physics Letters, April 2016.

[5] El-Hajje, G. et al. "Contactless Characterization Of Metastable Defects In $\mathrm{Cu}(\mathrm{In}, \mathrm{Ga}) \mathrm{Se} 2$ Solar Cells Using Time-Resolved Photoluminescence". Solar Energy Materials and Solar Cells 145 (2016): 462-467.

[6] Cao, Qing et al. "Defects In Chalcopyrite Semiconductors: Defects In $\mathrm{Cu}(\mathrm{In}, \mathrm{Ga}) \mathrm{Se} 2$ Chalcopyrite Semiconductors: A Comparative Study Of Material Properties, Defect States, And Photovoltaic Performance". Advanced Energy Materials 1.5 (2011): 844-844.
[7] Delamarre, Amaury et al. "Quantitative Luminescence Mapping Of $\mathrm{Cu}(\mathrm{In}, \mathrm{Ga}) \mathrm{Se} 2$ Thin-Film Solar Cells". Prog. Photovolt: Res. Appl. 23.10 (2014): 1305-1312. 